\title{
A polifarmácia entre pacientes hipertensos e diabéticos em uma unidade de saúde
}

\author{
Polypharmacy between hypertensive and diabetic patients in a health unit \\ Polifarmacia entre hipertensos y diabéticos en una unidad de salud
}

Adriana Conceição Borges da Silva1*, Bárbara Caroline Garcia Freitas ${ }^{1}$, Bruna Ferreira de Carvalho $^{1}$, Eluélly Lorrane da Conceição Rodrigues ${ }^{1}$, Fabyanne Silva de Oliveira ${ }^{1}$, lasmin Vasconcelos da Costa ${ }^{1}$, Eliane Leite da Trindade ${ }^{1,2}$.

\section{RESUMO}

Objetivo: Avaliar a presença de polifarmácia e interações medicamentosas entre pacientes hipertensos e diabéticos em uma unidade de saúde no estado do Pará. Métodos: Este estudo foi dividido em duas etapas: a primeira consistiu na aplicação de um questionário, avaliando variáveis sociodemográficas, clínicas e informações de polifarmácia; na segunda, foram detectados a presença de interações medicamentosas através do sistema Drug Interaction Checker. Resultados: Participaram do estudo 101 pacientes hipertensos e/ou diabéticos atendidos na Unidade Básica de Saúde do estado do Pará. A maioria foram pacientes mulheres $(69,3 \%)$, com faixa etária superior ou igual aos 60 anos $(67,3 \%)$ e com renda menor ou igual a um salário mínimo (51,5\%). 41,6\% ( $n=42)$ praticavam a utilização de 5 ou mais medicamentos. Foi identificado interações medicamentosas em $76 \%$ dos pacientes atendidos $(n=77)$ e em $24 \%(n=24)$ não foi identificado qualquer interação. O programa Drug Interaction Checker identificou 237 tipos de interações medicamentosas, sendo 7\% grave, $72 \%$ moderada e $21 \%$ leve. Conclusão: Enfatiza-se a necessidade de orientação aos pacientes acompanhados por doenças crônicas devido à complexidade do tratamento e a grande probabilidade de ocorrência de interações medicamentosas.

Palavras-chave: Polifarmácia, Interações medicamentosas, Diabetes mellitus, Hipertensão.

\begin{abstract}
Objective: To evaluate the presence of polypharmacy and drug interactions between hypertensive and diabetic patients in a health unit in the state of Pará. Methods: This study was divided into two stages: the first consisted of the application of a questionnaire, evaluating sociodemographic, clinical and polypharmacy information; in the second, the presence of drug interactions through the Drug Interaction Checker system was detected. Results: The study included 101 hypertensive and/or diabetic patients treated at the Basic Health Unit in the state of Pará. Most were female patients (69.3\%), aged 60 years or older (67.3\%) and with income less than or equal to a minimum wage $(51.5 \%) .41 .6 \%(n=42)$ practiced the use of 5 or more medications. Drug interactions were identified in $76 \%$ of patients seen $(n=77)$ and in $24 \%(n=24)$ no interactions were identified. The Drug Interaction Checker program identified 237 types of drug interactions, 7\% severe, 72\% moderate and $21 \%$ mild. Conclusion: The need for guidance to patients accompanied by chronic diseases is emphasized due to the complexity of the treatment and the high probability of occurrence of drug interactions.
\end{abstract}

Key words: Polypharmacy, Drug interactions, Diabetes mellitus, Hypertension.

1 Universidade do Estado do Pará (UEPA), Belém - PA. *E-mail: adriana.csilva@aluno.uepa.br

2 Universidade da Amazônia (UNAMA), Belém - PA.

SUBMETIDO EM: 6/2021

ACEITO EM: 6/2021

PUBLICADO EM: 8/2021 


\section{RESUMEN}

Objetivo: Evaluar la presencia de polifarmacia e interacciones medicamentosas entre pacientes hipertensos y diabéticos en una unidad de salud en el estado de Pará. Métodos: Este estudio se dividió en dos etapas: la primera consistió en la aplicación de un cuestionario, evaluando información sociodemográfica, clínica y polifarmacia; en el segundo, se detectó la presencia de interacciones medicamentosas a través del sistema Drug Interaction Checker. Resultados: El estudio incluyó a 101 pacientes hipertensos y/o diabéticos atendidos en la Unidad Básica de Salud del estado de Pará, la mayoría eran pacientes del sexo feminino $(69,3 \%)$, de 60 años o más $(67,3 \%)$ y con ingresos inferiores o iguales al salario mínimo (51,5\%). El 41,6\% $(\mathrm{n}=42)$ tomaba 5 o más medicamentos. Se identificaron interacciones farmacológicas en el $76 \%$ de los pacientes atendidos $(n=77)$, mientras que el $24 \%(n=24)$ se identificaron libres de estas interacciones. El programa Drug Interaction Checker detectó 237 tipos de interacciones medicamentosas, 7\% graves, 72\% moderadas y $21 \%$ leves. Conclusión: Se enfatiza la necesidad de orientación a los pacientes pluripatológicos debido a la complejidad del tratamiento y a la alta probabilidad de presentar interacciones medicamentosas.

Palabras clave: Polifarmacia, Interacciones con las drogas, Diabetes mellitus, Hipertensión.

\section{INTRODUÇÃO}

A transição demográfica que o Brasil está passando, com a elevação expressiva do número de idosos, tem contribuído para o aumento de casos de doenças crônico-degenerativas, com quadros medicamentosos cada vez mais complexos. Dentre estas destacam-se o Diabetes Mellitus (DM) e a Hipertensão Arterial Sistêmica (HAS), pois são as doenças mais relacionadas ao uso crônico de medicamentos e a prática da polifarmácia (CORRALO VS, et al., 2018; SANTOS JS, et al., 2019).

Nesse contexto, HAS e DM, classificadas como doenças crônicas não transmissíveis (DCNT), são tratadas como graves problemas de saúde, no Brasil, correspondendo a um elevado número de mortes prematuras e perda de qualidade de vida, além do impacto econômico para as sociedades e sistemas de saúde. Nessa perspectiva, o tratamento medicamentoso ainda se constitui como a principal prática que atua no controle e terapêutica dessas patologias. Assim, proporcionar cuidados básicos na prescrição e monitoramento desses fármacos se torna um desafio da saúde pública atual (STOPA SR, et al., 2018).

O conceito de polifarmácia foi inicialmente discutido no ano de 1959 e, a partir disso, surgiram diversas pesquisas que abordavam esse assunto. Assim sendo, de acordo com a literatura atual, a polifarmácia ou polimedicação é definida como o uso de cinco ou mais medicamentos simultaneamente durante o período equivalente a uma semana (CORRALO VS, et al., 2018). Os medicamentos mais comuns entre diabéticos e hipertensos são anti-hipertensivos, antidiabéticos, antidislipidémicos, antitrombóticos, hipoglicemiantes e outros. Nesse cenário, o grande número de medicamentos utilizados pode favorecer o surgimento de interações medicamentosas (IM) (KHAJA KAA, et al., 2018).

A IM, por sua vez, é um evento clínico em que os efeitos de um fármaco são alterados pela presença de uma ou mais substâncias, que pode ser outro fármaco, fitoterápico, alimento, bebida, entre outros. E as respostas consequentes dessas associações nem sempre são benéficas e podem ocasionar a potencialização do efeito terapêutico de um medicamento ou redução da sua eficácia, bem como o surgimento de reações adversas, afetando o tratamento do paciente e colocando em risco a sua qualidade de vida (ALVES NR, et al., 2019; LUZ V, et al 2018).

Desse modo, o processo de acompanhamento farmacoterapêutico, especialmente de pacientes idosos, é de extrema importância para o alcance do uso racional de medicamentos, contribuindo no processo educativo de seus consumidores, a fim de que estes possam ter um melhor entendimento sobre a complexidade de um regime posológico e adesão a determinado tipo de tratamento (RODRIGUES MCAS, et al., 2016).

Assim, o objetivo desse trabalho foi investigar a ocorrência da polifarmácia e seus efeitos colaterais em diabéticos e hipertensos atendidos em uma unidade de saúde no estado do Pará. 


\section{MÉTODOS}

Foi realizado um estudo transversal e quantitativo, com análise estatística descritiva, utilizando dados de pacientes diabéticos e/ou hipertensos, que realizavam acompanhamento pelo Sistema de Cadastramento e Acompanhamento de Portadores de Hipertensão Arterial e/ou Diabetes Mellitus (HIPERDIA), em uma unidade de saúde, no estado do Pará, durante os meses de julho a setembro de 2019.

O questionário foi aplicado somente após a assinatura do Termo de Consentimento Livre e Esclarecido (TCLE) pelos pacientes que aceitaram participar do estudo. Esse projeto recebeu a aprovação do Comitê de Ética em Pesquisa Envolvendo Seres Humanos da Universidade do Estado do Pará (UEPA)/ Centro de Ciências Biológicas e da Saúde, por meio do parecer consubstanciado de número 3.516.298/2019.

Para os critérios de inclusão dos candidatos a participarem do estudo, foram considerados apenas adultos (maiores de 18 anos), diagnosticados com HAS e/ou DM, que realizavam acompanhamento através do sistema HIPERDIA em uma unidade de saúde do Pará. Foram excluídos da pesquisa menores de idade e pacientes que não aceitaram participar do estudo.

Para a obtenção dos dados envolvendo a polifarmácia e IM, a pesquisa envolveu duas etapas, sendo a primeira a aplicação dos questionários aos pacientes hipertensos e/ou diabéticos, e a segunda a identificação de IM nesses mesmos pacientes, através de um software on-line.

A primeira etapa do estudo consistiu na aplicação de um questionário aos pacientes, em que foram avaliados variáveis sociodemográficas e clínicas de diabéticos e/ou hipertensos atendidos em uma unidade de saúde do Pará. Das variáveis sociodemográficas foram considerados: sexo (feminino ou masculino), faixa etária (a partir de 18 anos) e renda ( $\leq 1$ salário mínimo ou >1 salário mínimo). Para as variáveis clínicas foram avaliados: doença de base (DM, HAS ou os dois), tempo de doença, tempo em que participa do sistema HIPERDIA, informações de polifarmácia e automedicação.

Sobre os medicamentos, tanto os receitados como os de automedicação, foram levados em conta informações sobre sua forma farmacêutica e finalidade terapêutica. Em casos de automedicação, remédios de origem caseira foram desconsiderados do estudo. Além disso, os participantes envolvidos, também, foram questionados quanto aos cuidados básicos em relação ao uso de seus medicamentos, nesse caso o horário correto de administração, período de tratamento e indicação por próximos (parentes, vizinhos, amigos).

$\mathrm{Na}$ segunda etapa do estudo, para estimar a presença de IM, foram identificados os medicamentos consumidos por todos os pacientes nos últimos 7 dias. Esses medicamentos, então, foram inseridos em um programa de software on-line, o Drug Interaction Checker, que realiza uma pesquisa bibliográfica em três bancos de dados: US Food and Drug Administration (FDA), American Hospital Formulary Service (AHFS Drug Information) e First Data Bank, gerando um relatório contendo as seguintes informações: presença de interação, fármacos envolvidos e a severidade (MEDSCAPE, 2020).

Sobre a severidade das interações, estas foram classificadas como grave (a combinação de fármacos está contraindicada e deve ser dispensada ao paciente), moderada (medidas de ação são necessárias para diminuir o risco de interação) e leve (o risco de interação deve ser avaliado e medidas de ação também são necessárias). Além disso, o sistema fornece uma pequena explicação sobre os mecanismos de ação dos medicamentos envolvidos.

Para apresentação dos resultados, os pacientes foram listados em três grupos de estudo (hipertensos, diabéticos, hipertensos e diabéticos). Os dados coletados, através do questionário e do programa Drug Interaction Checker, foram registrados no Microsoft Office Excel 2018, a fim de se realizar a avaliação das frequências quantitativas do grupo amostral envolvido no estudo.

\section{RESULTADOS}

Participaram do estudo 101 pacientes hipertensos e/ou diabéticos, sendo que 13 eram apenas diabéticos, 56 eram apenas hipertensos e 32 possuíam as duas doenças. Sobre o perfil sociodemográfico total desses participantes, em sua maioria, eles pertenciam ao sexo feminino $(69,3 \%)$, possuíam idade superior ou igual 
aos 60 anos (67,3\%) e recebiam até um salário mínimo (51,5\%). Deste total, 29,7\% possuíam doença entre um e cinco anos e cerca de $29,7 \%$ possuíam tempo de acompanhamento médico equivalente a este período (Tabela 1).

Tabela 1 - Perfil sociodemográfico dos pacientes com hipertensão e/ou Diabetes atendidos pelo sistema HIPERDIA de uma unidade de saúde do Pará.

\begin{tabular}{|c|c|c|c|c|c|c|c|c|}
\hline \multirow[t]{2}{*}{ Variável } & \multicolumn{2}{|c|}{ Diabetes } & \multicolumn{2}{|c|}{ Hipertensão } & \multicolumn{2}{|c|}{$\begin{array}{l}\text { Hipertensão/ } \\
\text { Diabetes }\end{array}$} & \multicolumn{2}{|c|}{ Total Geral } \\
\hline & $\mathbf{N}$ & $\%$ & $\mathbf{N}$ & $\%$ & $\mathbf{N}$ & $\%$ & $\mathbf{N}$ & $\%$ \\
\hline \multicolumn{9}{|l|}{ Sexo } \\
\hline Masculino & 3 & 23,1 & 20 & 35,7 & 24 & 75,0 & 31 & 30,7 \\
\hline Feminino & 10 & 76,9 & 36 & 64,3 & 8 & 25,0 & 70 & 69,3 \\
\hline \multicolumn{9}{|l|}{ Idade } \\
\hline 30 a 59 & 7 & 53,8 & 17 & 30,4 & 9 & 28,1 & 33 & 32,7 \\
\hline$\geq 60$ anos & 6 & 46,2 & 39 & 69,6 & 23 & 71,9 & 68 & 67,3 \\
\hline \multicolumn{9}{|l|}{ Renda } \\
\hline$\leq 1$ salário mínimo & 9 & 69,2 & 27 & 48,2 & 16 & 50,0 & 52 & 51,5 \\
\hline > 1 salário mínimo & 4 & 30,8 & 23 & 41,1 & 10 & 31,3 & 37 & 36,6 \\
\hline Não soube & 0 & 0,0 & 6 & 10,7 & 6 & 18,8 & 12 & 11,9 \\
\hline \multicolumn{9}{|l|}{ Tempo doença } \\
\hline$<1$ ano & 1 & 7,7 & 0 & 0 & 6 & 18,8 & 7 & 6,9 \\
\hline $1-5$ anos & 6 & 46,2 & 13 & 23,2 & 11 & 34,4 & 30 & 29,7 \\
\hline $6-10$ anos & 2 & 15,4 & 14 & 25,0 & 10 & 31,3 & 26 & 25,7 \\
\hline $11-20$ anos & 1 & 7,7 & 19 & 33,9 & 5 & 15,6 & 25 & 24,8 \\
\hline$>20$ anos & 3 & 23,1 & 10 & 17,9 & 0 & 0,0 & 13 & 12,9 \\
\hline \multicolumn{9}{|l|}{ Tempo de tratamento } \\
\hline$<1$ ano & 3 & 23,1 & 10 & 17,9 & 6 & 18,8 & 19 & 18,8 \\
\hline $1-5$ anos & 5 & 38,5 & 14 & 25,0 & 11 & 34,4 & 30 & 29,7 \\
\hline $6-10$ anos & 2 & 15,4 & 13 & 23,2 & 10 & 31,3 & 25 & 24,8 \\
\hline $11-20$ anos & 3 & 23,1 & 13 & 23,2 & 5 & 15,6 & 21 & 20,8 \\
\hline$>20$ anos & 0 & 0,0 & 6 & 10,7 & 0 & 0,0 & 6 & 5,9 \\
\hline
\end{tabular}

Fonte: Silva ACB, et al., 2021.

Com base nas respostas do questionário, 98 pacientes (97\%) faziam uso regular das medicações, sendo 11 do grupo de diabéticos, 55 do grupo de hipertensos e 32 do grupo de diabéticos e hipertensos, porém a maioria $(92,1 \%)$ relatou ter prescrições médicas para essa finalidade. Sobre a utilização de medicamentos para outras comorbidades, 54 indivíduos (53,5\%) responderam que não faziam tal uso. Além disso, 52,5\% não realizavam automedicação e $73,3 \%$ não faziam uso de fármacos por indicação de familiares ou amigos. Quanto à necessidade de utilização regular da medicação, 93,1\% tinham horários definidos para tal uso, mas cerca de $75,2 \%$ dos entrevistados relataram esquecer, pelo menos uma vez, de administrar seus 
medicamentos no período adequado. Ainda, $89,1 \%$ disseram não suspender a ingestão do medicamento após a melhora dos sintomas (Tabela 2).

Tabela 2 - Respostas do questionário aplicado aos participantes do sistema HIPERDIA de uma unidade de saúde do Pará.

\begin{tabular}{|c|c|c|c|c|c|c|c|c|c|c|}
\hline \multirow{3}{*}{ QUESTIONÁRIO } & \multirow{2}{*}{\multicolumn{2}{|c|}{$\begin{array}{l}\text { Diabetes } \\
\text { Mellitus }\end{array}$}} & \multirow{2}{*}{\multicolumn{2}{|c|}{ Hipertensão }} & \multirow{2}{*}{\multicolumn{2}{|c|}{$\begin{array}{l}\text { Hipertensão/ } \\
\text { Diabetes }\end{array}$}} & \multicolumn{4}{|c|}{ Total } \\
\hline & & & & & & & \multicolumn{2}{|c|}{ Sim } & \multicolumn{2}{|c|}{ Não } \\
\hline & Sim & Não & Sim & Não & Sim & Não & $\mathbf{N}$ & $\%$ & $\mathbf{N}$ & $\%$ \\
\hline $\begin{array}{l}\text { 1. Faz uso regular de } \\
\text { medicamentos para } \\
\text { tratamento de hipertensão } \\
\text { e/ou diabetes? }\end{array}$ & 11 & 2 & 55 & 1 & 32 & 0 & 98 & 97,0 & 3 & 3,0 \\
\hline $\begin{array}{l}\text { 2. Faz uso de } \\
\text { medicamentos para } \\
\text { tratamento de outras } \\
\text { doenças? }\end{array}$ & 7 & 6 & 25 & 31 & 15 & 17 & 47 & 46,5 & 54 & 53,5 \\
\hline $\begin{array}{l}\text { 3. Todos os medicamentos } \\
\text { que utiliza são prescritos } \\
\text { pelos médicos? }\end{array}$ & 12 & 1 & 50 & 6 & 31 & 1 & 93 & 92,1 & 8 & 7,9 \\
\hline $\begin{array}{l}\text { 4. Já fez o uso de } \\
\text { medicamentos por conta } \\
\text { própria? }\end{array}$ & 7 & 6 & 31 & 25 & 10 & 22 & 48 & 47,5 & 53 & 52,5 \\
\hline $\begin{array}{l}\text { 5. Já fez o uso de } \\
\text { medicamentos por } \\
\text { indicação de parentes e } \\
\text { amigos? }\end{array}$ & 5 & 8 & 18 & 38 & 4 & 28 & 27 & 26,7 & 74 & 73,3 \\
\hline $\begin{array}{l}\text { 6. Tem horário certo para } \\
\text { tomar os medicamentos? }\end{array}$ & 13 & 0 & 49 & 7 & 32 & 0 & 94 & 93,1 & 7 & 6,9 \\
\hline $\begin{array}{l}\text { 7. Já esqueceu de tomar } \\
\text { os medicamentos? }\end{array}$ & 10 & 3 & 42 & 14 & 24 & 8 & 76 & 75,2 & 25 & 24,8 \\
\hline $\begin{array}{l}\text { 8. Quando se sente } \\
\text { melhor para de tomar os } \\
\text { medicamentos por conta } \\
\text { própria? }\end{array}$ & 2 & 11 & 7 & 49 & 2 & 30 & 11 & 10,9 & 90 & 89,1 \\
\hline
\end{tabular}

Fonte: Silva ACB, et al., 2021.

Em relação aos medicamentos utilizados, $58,4 \%$ dos pacientes não consumiam mais de 5 medicamentos. Entretanto, $41,6 \%$ praticavam polifarmácia, entre eles 30,8\% indivíduos do grupo de diabéticos, $41,1 \%$ dos hipertensos e $46,9 \%$ dos pacientes com DM e HAS. Estavam sob terapêutica médica, $34,7 \%(n=35)$ dos pacientes que consumiam mais de 5 medicamentos, bem como 62,4\% ( $n=63)$ daqueles que consumiam de um a quatro medicamentos. Já sobre a automedicação, apenas um total de $24,8 \%$ dos pacientes $(n=25)$ realizavam tal prática (Figura 1). 
Figura 1 - Percentual de pacientes atendidos pelo sistema HIPERDIA que praticam polifarmácia.

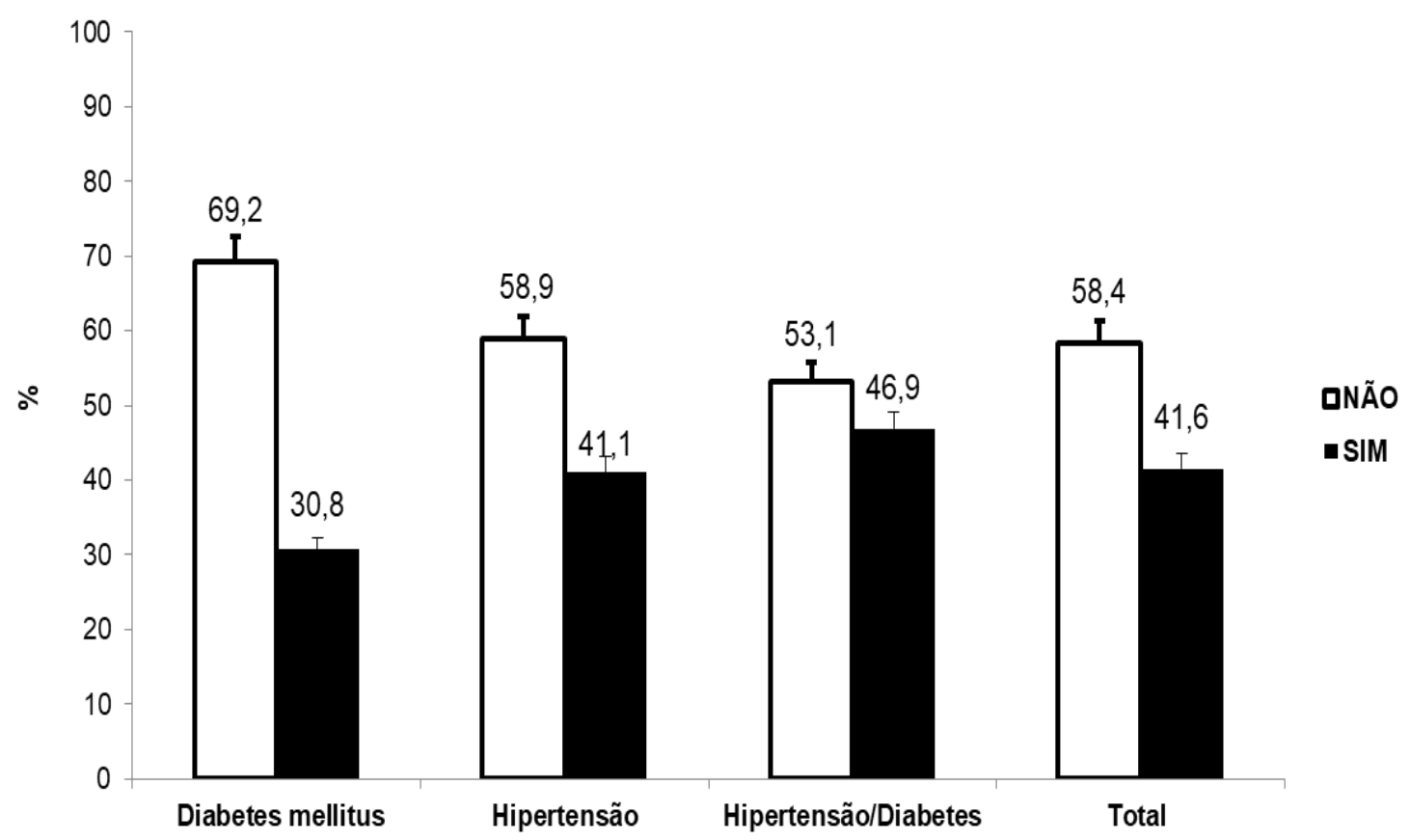

Fonte: Silva ACB, et al., 2021.

Em relação aos pacientes atendidos pelo sistema HIPERDIA de uma unidade de saúde do Pará, em 77 indivíduos foi identificado IM, variando entre leve ( $21 \%$ ou $n=49)$, moderada $(72 \%$ ou $n=171)$ e grave $(7 \%$ ou $\mathrm{n}=17$ ). Entre pacientes com DM, identificou-se um total de 31 tipos de IM, sendo a combinação dos medicamentos prometazina e o risperidona (13\%) a mais visualizada. A metformina, apareceu em duas interações das cinco listadas na tabela. Já a interação de nível mais grave foi entre o carbamazepina e o diazepam (Tabela 3).

Tabela 3 - Interações Medicamentosas encontradas em Pacientes com Diabetes Mellitus.

\begin{tabular}{|c|c|c|c|c|}
\hline Medicamento & $\begin{array}{l}\text { Tipo de } \\
\text { Interação }\end{array}$ & Explicação & $\mathbf{N}$ & $\%$ \\
\hline $\begin{array}{l}\text { Prometazina }+ \\
\text { Risperidona }\end{array}$ & Moderada & $\begin{array}{l}\text { Os moduladores de serotonina podem aumentar o } \\
\text { bloqueio da dopamina, possivelmente aumentando o } \\
\text { risco de síndrome maligna dos neurolépticos. Os } \\
\text { antipsicóticos podem aumentar o efeito serotoninérgico } \\
\text { dos moduladores da serotonina, o que pode resultar na } \\
\text { síndrome da serotonina. }\end{array}$ & 4 & 13,0 \\
\hline $\begin{array}{l}\text { Celecoxib + } \\
\text { Diclofenaco }\end{array}$ & Moderada & Celecoxibe e diclofenaco aumentam a anticoagulação. & 3 & 9,7 \\
\hline $\begin{array}{l}\text { Hidroclorotiazida + } \\
\quad \text { Metformina }\end{array}$ & Moderada & $\begin{array}{l}\text { A hidroclorotiazida pode aumentar os níveis de açúcar } \\
\text { no sangue e interferir no controle do diabetes. }\end{array}$ & 2 & 6,5 \\
\hline $\begin{array}{l}\text { Carbamazepina }+ \\
\quad \text { Diazepam }\end{array}$ & Grave & $\begin{array}{l}\text { A carbamazepina diminui o nível ou o efeito do } \\
\text { diazepam, afetando o metabolismo da enzima } \\
\text { hepática/intestinal. }\end{array}$ & 2 & 6,5 \\
\hline $\begin{array}{l}\text { Levotiroxina }+ \\
\text { Metformina }\end{array}$ & Moderada & $\begin{array}{l}\text { Levotiroxina diminui os efeitos da metformina por } \\
\text { antagonismo farmacodinâmico. }\end{array}$ & 2 & 6,5 \\
\hline Total & - & & 31 & 100,0 \\
\hline
\end{tabular}

Fonte: Silva ACB, et al., 2021. 
Em pacientes com HAS, identificou-se 229 tipos de IM, sendo as combinações de losartana e aspirina $(7,9 \%)$ e losartana e atenolol $(7,9 \%)$ as mais prevalentes (Tabela 4$)$.

Tabela 4 - Interações Medicamentosas encontradas em Pacientes com Hipertensão Arterial.

\begin{tabular}{|c|c|c|c|c|}
\hline Medicamento & $\begin{array}{l}\text { Tipo de } \\
\text { Interação }\end{array}$ & Explicação & $\mathbf{N}$ & $\%$ \\
\hline $\begin{array}{l}\text { Losartana }+ \\
\text { Aspirina }\end{array}$ & Moderada & $\begin{array}{l}\text { Aumentam a toxicidade um do outro. Pode resultar em } \\
\text { deterioração da função renal, particularmente em idosos } \\
\text { ou em indivíduos com depleção de volume. }\end{array}$ & 18 & 7,9 \\
\hline $\begin{array}{l}\text { Losartana }+ \\
\text { Atenolol }\end{array}$ & Moderada & Aumentam o potássio sérico. & 18 & 7,9 \\
\hline $\begin{array}{l}\text { Losartana }+ \\
\text { Hidroclorotiazida }\end{array}$ & Moderada & $\begin{array}{l}\text { A losartana aumenta e a hidroclorotiazida diminui o } \\
\text { potássio sérico. Efeito da interação não é claro. }\end{array}$ & 17 & 7,4 \\
\hline $\begin{array}{l}\text { Aspirina }+ \\
\text { Hidroclorotiazida }\end{array}$ & Leve & $\begin{array}{l}\text { A aspirina aumenta e a hidroclorotiazida diminui o } \\
\text { potássio sérico. }\end{array}$ & 12 & 5,2 \\
\hline $\begin{array}{l}\text { Losartana }+ \\
\text { Diclofenaco }\end{array}$ & Moderada & $\begin{array}{l}\text { Aumentam a toxicidade um do outro. Pode resultar em } \\
\text { deterioração da função renal, particularmente em idosos } \\
\text { ou em indivíduos com depleção de volume. }\end{array}$ & 6 & 2,6 \\
\hline Total & - & & 229 & 100,0 \\
\hline
\end{tabular}

Fonte: Silva ACB, et al., 2021.

Já em pacientes com ambas comorbidades, registrou-se 193 tipos de IM, sendo as associações de losartana e atenolol $(7,3 \%)$ e hidroclorotiazida e metformina $(7,3 \%)$ as mais predominantes (Tabela 5).

Tabela 5 - Interações Medicamentosas encontradas em Pacientes com Diabetes Mellitus e Hipertensão Arterial.

\begin{tabular}{|c|c|c|c|c|}
\hline Medicamento & $\begin{array}{l}\text { Tipo de } \\
\text { Interação }\end{array}$ & Explicação & $\mathbf{N}$ & $\%$ \\
\hline $\begin{array}{l}\text { Losartana }+ \\
\quad \text { Atenolol }\end{array}$ & Moderada & Aumentam o potássio sérico. & 14 & 7,3 \\
\hline $\begin{array}{l}\text { Hidroclorotiazida } \\
+ \text { Metformina }\end{array}$ & Moderada & $\begin{array}{l}\text { A hidroclorotiazida pode aumentar os níveis de } \\
\text { açúcar no sangue e interferir no controle do diabetes. }\end{array}$ & 14 & 7,3 \\
\hline $\begin{array}{l}\text { Losartana }+ \\
\text { Hidroclorotiazida }\end{array}$ & Moderada & $\begin{array}{l}\text { A losartana aumenta e a hidroclorotiazida diminui o } \\
\text { potássio sérico. Efeito da interação não é claro. }\end{array}$ & 9 & 4,7 \\
\hline $\begin{array}{l}\text { Losartana }+ \\
\text { Aspirina }\end{array}$ & Moderada & $\begin{array}{l}\text { Aumentam a toxicidade um do outro. Pode resultar } \\
\text { em deterioração da função renal, particularmente em } \\
\text { idosos ou em indivíduos com depleção de volume. }\end{array}$ & 6 & 3,1 \\
\hline $\begin{array}{l}\text { Losartana + } \\
\text { Insulina }\end{array}$ & Moderada & $\begin{array}{l}\text { A losartana aumenta os efeitos da insulina humana } \\
\text { normal por mecanismo de interação não } \\
\text { especificado. O uso concomitante de insulina e } \\
\text { losartana pode requerer ajuste da dose de insulina e } \\
\text { maior monitoramento da glicose. }\end{array}$ & 5 & 2,6 \\
\hline Total & - & & 193 & 100,0 \\
\hline
\end{tabular}

Fonte: Silva ACB, et al., 2021. 


\section{DISCUSSÃO}

A prevalência do uso de medicamentos para doenças crônicas no Brasil é elevada, contribuindo também para o estabelecimento da polifarmácia (TAVARES NUL, et al., 2015). Neste estudo, a frequência encontrada de polifarmácia foi de $41,6 \%$ entre os pacientes hipertensos e/ou diabéticos, que foi superior ao achado de outro estudo realizado entre pacientes atendidos por uma Estratégia Saúde da Família no Mato Grosso do Sul, em que apenas $20 \%$ dos indivíduos se apresentavam nessa condição. A polifarmácia nem sempre é um evento evitável, pois em casos de doenças crônicas, como HAS e DM, é necessário a administração de diversos medicamentos para controle de tais patologias, porém é importante enfatizar que a prescrição desses fármacos ocorra de forma adequada, garantindo a segurança dos pacientes (FREITAS AFK, et al., 2021).

Sobre o perfil sociodemográfico, observou-se a predominância de idosos acima dos 60 anos (67,3\%) e do sexo feminino $(69,3 \%)$. Tal achado pode ser explicado pela medicalização excessiva dentro desta faixa etária, uma vez que, durante o envelhecimento, é mais comum o aparecimento de doenças crônicas (SIMONETTI $A B$, et al., 2021). Já em relação ao predomínio do sexo feminino, isso pode estar associado a própria amostra estudada, composta majoritariamente por mulheres. De outro modo, estudos também apontam que as mulheres em idade avançada são mais acometidas por doenças crônicas não fatais, no Brasil, necessitando, então, do controle medicamentoso (CORRALO VS, et al., 2018).

Em relação a renda mensal, a maioria dos pacientes possuíam renda menor ou igual a um salário mínimo $(51,5 \%)$. Tal achado, também, pode ser associado à baixa prática da automedicação neste grupo de estudo, visto que a aquisição de outros medicamentos mais caros se torna difícil, fazendo com que este público utilize apenas os fármacos fornecidos pelo Sistema Único de Saúde (SUS) para o tratamento de doenças crônicas, como HAS e DM (BARRETO MNSC, et al., 2015).

Acerca das variáveis clínicas "tempo de doença" e "tempo de tratamento", os dados obtiveram semelhanças entre a maior parte dos pacientes diabéticos e com as duas comorbidades (hipertensos/diabéticos), com períodos de 1 a 5 anos; diferente dos hipertensos, que apresentaram uma disparidade, onde o tempo de doença (11 a 20 anos) foi maior que o tempo de tratamento (1 a 5 anos). Esse achado pode ser associado ao curso assintomático da própria HAS, uma vez que, por não haver a manifestação de sintomas, as pessoas acometidas por essa doença não buscam seu tratamento precoce, influenciando, também, no agravamento dessa patologia e surgimento de possíveis complicações (SILVA MGC, et al., 2018).

No estudo, também foi possível notar que os pacientes hipertensos se apresentaram mais negligentes quanto ao horário certo para administrar seus medicamentos $(n=42)$, bem como ao deixar de tomá-los em função, de não só esquecer, mas também, de melhoras a curto prazo $(n=7)$. Esse dado, equipara-se ao estudo de Ramos LR, et al. (2016), o qual afirma que esta prática, especialmente adotada pelo grupo de hipertensos, interfere diretamente no grau de adesão ao tratamento por essas pessoas.

Sobre a prática da polifarmácia, comparando os pacientes que faziam uso constante de medicações para DM e HAS, observou-se uma maior presença da polifarmácia em pacientes com as duas comorbidades (46,9\%). Esses dados denotam que o aparecimento de comorbidades somado a doenças crônicas são fatores que levam a uma prevalência maior da polifarmácia, haja em vista a necessidade do uso de vários medicamentos para controlar tais doenças (NASCIMENTO RCRM, et al., 2017). Já a HAS foi a segunda patologia que apresentou o maior grau de polifarmácia $(41,1 \%)$. Essa doença, por sua vez, atinge aproximadamente 30 milhões de brasileiros e cerca de 50\% destes são assintomáticos. Desse modo, caracteriza-se como o fator de risco modificável mais bem relacionado com a morbimortalidade cardiovascular e a principal responsável pela redução da sobrevida em diabéticos (CORRALO VS, et al., 2018).

Também foi possível evidenciar que quase todos os pacientes utilizavam apenas os medicamentos prescritos pelo médico $(92,1 \%)$ e a maioria deles não consumiam outros fármacos $(53,5 \%)$ se não para o tratamento de HAS e DM. Do mesmo modo, notou-se que boa parte daqueles que se encontravam em situação de polifarmácia $(41,6 \%)$, estavam sob terapêutica médica $(34,7 \%)$. Nesse sentido, é notória a ausência de evidências para apoiar as decisões de prescrição, haja vista o baixo número de ensaios clínicos envolvendo estudos sobre IM (BROEKHUIZEN K, et al., 2015). 
Além disso, possível evidenciar que a ocorrência de IM entre os pacientes foi proporcional a quantidade de medicamentos prescritos, o que reforça a necessidade de uma melhor avaliação da prescrição de fármacos e orientação para o seu uso racional, principalmente, entre indivíduos idosos e com várias comorbidades, pois é comum se observar a utilização de diversos compostos com o mesmo princípio ativo ou finalidade terapêutica, o que, por vezes, não beneficia o tratamento do paciente e até pode colocar em risco a sua qualidade de vida (SOUZA DM, et al., 2018).

Sobre dados de automedicação, notou-se uma prevalência de $24,8 \%$ entre os pacientes hipertensos e/ou diabéticos e a maioria relatou não fazer uso de medicamentos por conta própria (52,5\%) ou por indicação de terceiros $(73,3 \%)$. Outros estudos também demostraram que há uma baixa prática de automedicação, especialmente, entre idosos e com doenças crônicas, haja em vista que, por estarem em constante acompanhamento médico, provavelmente não dependem dessa prática para seus tratamentos terapêuticos (DOMINGUES PHF, et al., 2017).

Acerca das IM, neste trabalho foi observado a maior frequência de IM de risco moderado (72\%), seguido de risco leve $(21 \%)$ e posteriormente de risco grave $(7 \%)$. Esse dado revela a necessidade de acompanhamento e monitoramento desses casos, pois os riscos da interação entre dois fármacos, se visualizados isoladamente, podem não sugerir grandes perigos, mas quando se trata de casos de polifarmácia, as interações tendem a aumentar e se estabelecer a longo prazo, prejudicando a saúde e o tratamento dos pacientes (REMPEL C, et al., 2015).

Ao avaliar os três grupos de estudo, foram observados uma maior frequência de IM em pacientes com HAS ( $n=229)$ e HAS e DM ( $n=193)$, levando-se em consideração a maior quantidade de fármacos empregados no tratamento de tais comorbidades, como exposto anteriormente.

A interação mais frequentemente encontrada nos pacientes com HAS foi entre losartana e aspirina $(7,9 \%)$ e losartana e atenolol (7,9\%). A losartana potássica é um anti-hipertensivo da classe dos bloqueadores do receptor da angiotensina II (BRAs), que atua no controle da pressão arterial e pode ser associado a outros medicamentos, como o ácido acetilsalić́lico, denominado de aspirina (evita a ocorrência de infarto), e outros anti-hipertensivos, como o atenolol (betabloqueador seletivo que atua no controle da arritmia cardíaca). Contudo, em ambos os casos, o risco de interação é moderado, o que revela a necessidade de monitoramento constante dessas associações, pois o uso prolongado desses fármacos pode aumentar a toxicidade no organismo e prejudicar a função renal (FARIA ALG, et al., 2019).

No grupo com DM e HAS, as interações entre losartana e atenolol (7,3\%) e hidroclorotiazida e metformina $(7,3 \%)$ foram as mais predominantes. Assim como nos pacientes com HAS, a associação de losartana e atenolol também é muito comum, por motivos já explicitados anteriormente. Já a interação entre hidroclorotiazida (da classe dos diuréticos) e cloridrato metformina (possui efeito anti-hiperglicêmico), por ser de risco moderado, requer constante monitoramento, uma vez que a hidroclorotiazida possui efeito hiperglicemiante e pode interferir no controle da glicose no sangue (ALVES NR, et al., 2019).

Dentre os pacientes com DM, a interação mais frequentemente encontrada foi entre a prometazina e risperidona (13\%) o que diverge dos resultados apresentados por Rempel C, et al. (2015), que afirma em sua pesquisa ser glibenclamida e metformina. Tal fato pode estar relacionado a pequena amostra de pacientes diabéticos analisada em nosso estudo $(n=13)$ e a maioria destes faziam o consumo de medicamentos para outras doenças $(n=7)$.

A associação de prometazina (anti-histamínico) e risperidona (antipsicótico) é considerado de risco moderado, visto que a interação desses medicamentos pode fazer com que os moduladores de serotonina elevem a ação de bloqueio da dopamina, eventualmente, ampliando o risco de síndrome maligna dos neurolépticos, em que o paciente pode apresentar estado mental alterado, anormalidades motoras, hipertermia $\left(>38^{\circ} \mathrm{C}\right)$ e hiperatividade de movimentos autonômicos. Por outro lado, os antipsicóticos podem ampliar o efeito serotoninérgico dos moduladores de serotonina, desenvolvendo a síndrome da serotonina, que também inclui manifestações clínicas graves, como hipertermia e atividade muscular excessiva (AZOFEIFA MM, et al., 2019; CHAVARRÍA MJO, et al., 2019). 
Em vista disso, a aplicação de estratégias, que visem o aprimoramento das prescrições médicas e a conscientização da população sobre consumo adequado de medicamentos, são de suma importância para atenuar os danos gerados pela polifarmácia e a prática da automedicação (NASCIMENTO RCRM, et al., 2017). Nesse sentido, a discussão entre profissionais da saúde para elaboração de protocolos de controle de medicamentos, bem como o desenvolvimento de atividades de educação em saúde são formas de amenizar os riscos associados as IM, pois têm o intuito de promover o uso racional e controlado dos medicamentos, especialmente, para aqueles que recorrem ao sistema público de saúde (SILVA JS e DAMASCENA RS, 2017).

\section{CONCLUSÃO}

Participaram do estudo 101 pacientes hipertensos e/ou diabéticos atendidos na Unidade Básica de Saúde do estado do Pará. Destes, a maioria pertencia ao sexo feminino, com faixa etária superior ou igual aos 60 anos e com renda menor ou igual a um salário mínimo. Além disso, a maioria dos pacientes não praticavam polifarmácia e automedicação. Já sobre as interações medicamentosas, programa Drug Interaction Checker identificou 237 tipos e a maioria delas eram de risco moderado. Assim sendo, durante o nosso estudo foi possível observar a necessidade do acompanhamento aos pacientes, bem como, a atenção aos fármacos receitados e possíveis interações que podem decorrer disso. Desse modo, é importante enfatizar a necessidade de mais estudos de caráter farmacoepidemiológicos, especialmente, entre os pacientes acometidos por doenças crônicas, como hipertensão e diabetes mellitus, devido ao consumo exacerbado de medicamentos por esses indivíduos a longo prazo, o que aumenta, também, a probabilidade de ocorrência de interações medicamentosas.

\section{AGRADECIMENTOS}

Agradecemos a todos os pacientes que aceitaram participar do estudo e a equipe que trabalha no sistema HIPERDIA da unidade de saúde do estado do Pará pelo apoio na coleta de dados.

\section{REFERÊNCIAS}

1. ALVES NR, et al. Avaliação das interações medicamentosas entre anti-hipertensivos e hipoglicemiantes orais. Revista Multidisciplinar e de Psicologia, 2019; 13: 374-392.

2. AZOFEIFA MM, et al. Síndrome Serotoninérgico. Revista Clínica de la Escuela de Medicina, 2019; 9: 14-19.

3. BARRETO MNSC, et al. Análise do acesso ao tratamento medicamentoso para hipertensão e diabetes na Estratégia de Saúde da Família no Estado de Pernambuco, Brasil. Revista Brasileira de Epidemiologia, 2015; 18: 413-424.

4. BROEKHUIZEN K, et al. Characteristics of randomized controlled trials designed for elderly: a systematic review. Plos One, 2015; 10: 1-8.

5. CHAVARRÍA MJO, et al. Síndrome neuroléptico maligno. Acta Médica Grupo Ángeles, 2019; 17: $282-286$.

6. CORRALO VS, et al. Polifarmácia e fatores associados em idosos diabéticos. Revista de Salud Pública, 2018; 20 : 366-372.

7. DOMINGUES PHF, et al. Prevalência e fatores associados à automedicação em adultos no Distrito Federal: estudo transversal de base populacional. Epidemiologia e Serviços de Saúde, 2017; 26: 319-330.

8. FARIA ALG, et al. Avaliação das interações medicamentosas e possíveis efeitos colaterais em pacientes idosos da clínica cardiovascular. Diálogos Interdisciplinares, 2019; 8: 21-28.

9. FREITAS AFK, et al. Utilização de medicamentos por hipertensos e/ou diabéticos cadastrados em uma Estratégia Saúde da Família. Revista de Pesquisa Cuidado é Fundamental Online, 2021; 13: 57-64.

10. KHAJA KAA, et al. Potentially inappropriate prescribing in older adults with hypertension or diabetes mellitus and hypertension in a primary care setting in Bahrain. Medical Principles and Practice, 2018; 27: 241-249.

11. LUZ V, et al. Riscos de interações medicamentosas presentes nos receituários de pacientes hipertensos e diabéticos: uma revisão bibliográfica. Id on Line Revista Multidisciplinar e de Psicologia, 2018; 12: 793-806.

12. MEDSCAPE. Drug Interaction Checker. Disponível em: https://reference.medscape.com/drug-interactionchecker. Acessado em: 07 de maio de 2020.

13. NASCIMENTO RCRM, et al. Polifarmácia: uma realidade na atenção primária do Sistema Único de Saúde. Revista de Saúde Pública 2017; 51: 1-12.

14. RAMOS LR, et al. Polifarmácia e polimorbidade em idosos no Brasil: um desafio em saúde pública. Revista de Saúde Pública, 2016; 50: 1-13.

15. REMPEL C, et al. Análise da medicação utilizada por diabéticos e hipertensos. Revista Caderno Pedagógico, 2015; 12: $241-252$. 
16. RODRIGUES MCAS, et al. Perfil do consumo de medicamentos por frequentadores de centros de convivência de idosos. Revista Eletrônica Acervo Saúde, 2016; 8: 947-957.

17. SANTOS JS, et al. Interações medicamentosas potenciais em adultos e idosos na atenção primária. Ciência e Saúde Coletiva, 2019; 24: 4335-4344.

18. SILVA JS, DAMASCENA RS. Avaliação das interações medicamentosas potenciais no âmbito da UTI adulta. Id on Line Revista Multidisciplinar e de Psicologia, 2017; 11: 1-24.

19. SILVA MGC, et al. Hipertensão arterial e cuidados com a saúde: concepções de homens e mulheres. Psicologia, Saúde \& Doenças, 2018; 19: 435-452.

20. SIMONETTI $A B$, et al. Polifarmácia: prevalência e fatores associados em usuários da atenção primária à saúde de um município do sul do Brasil. Revista Eletrônica Acervo Saúde, 2021; 13: e7453.

21. SOUZA DM, et al. Uso inapropriado de medicamentos pelo idoso: polifarmácia e seus efeitos. Pensar Acadêmico, 2018; 16: 166-178.

22. STOPA SR, et al. Prevalência da hipertensão arterial, do diabetes mellitus e da adesão às medidas comportamentais no Município de São Paulo, Brasil, 2003-2015. Cadernos de Saúde Pública, 2018; 34: e00198717.

23. TAVARES NUL, et al. Uso de medicamentos para tratamento de doenças crônicas não transmissíveis no Brasil: resultados da Pesquisa Nacional de Saúde, 2013. Epidemiologia e Serviços de Saúde, 2015; 24: 315-323. 